\title{
STABLE HARMONIC MAPS INTO THE COMPLEX PROJECTIVE SPACES
}

\author{
JINGYI CHEN
}

\section{Introduction}

In this paper, we study smooth stable harmonic mappings from compact manifolds into the complex projective spaces. We begin with some general definitions. Let $(M, g)$ and $(N, h)$ be compact smooth Riemannian manifolds. A smooth map $f$ from $M$ into $N$ is harmonic if it is a critical point of the Dirichlet energy functional

$$
E(\phi)=\int_{M}|d \phi|^{2} d v_{g}
$$

where $\phi \in C^{\infty}(M, N)$, and $v_{g}$ denotes the Riemannian volume form of $(M, g)$. A harmonic map $f$ is stable if the second variation of the energy of $f$ is nonnegative, i.e., if

$$
0 \leq\left.\frac{d^{2}}{d t^{2}} E\left(f_{t}\right)\right|_{t=0}=2 \int_{M}\left(\left\|\nabla^{\prime} V\right\|^{2}-\sum_{\alpha}\left\langle R^{N}\left(V, f_{*} e_{\alpha}\right) V, f_{*} e_{\alpha}\right\rangle\right) d v_{g}
$$

for all smooth variations $f_{t}$ of $f$ with $f_{0}=f$, and the variational vector field $V=\left.\frac{\delta}{\delta t} f_{t}\right|_{t=0}$, where $\nabla^{\prime}$ denotes the pullback connection from $T N$ and $\left\{e_{\alpha}\right\}, \alpha=1, \ldots, n$, is an orthonormal basis for the tangent space of $M$. It is not hard to see that if the sectional curvature $R^{N}$ of $N$ is nonpositive, then every harmonic map is stable (in fact, minimizing) due to the convexity of the energy. But if $R^{N} \geq 0$, the energy functional fails to be convex in general, and a minimizing sequence may not converge. One result in this paper is that in many cases we cannot minimize energy by smooth maps in a given homotopy class.

Received November 18, 1993, and, in revised form, July 7,1994 . The author is partially supported by NSF grant \#9300422 
Another interesting aspect of the theory of harmonic maps is the theory of harmonic morphisms. Its history goes back to Jacobi in 1847 [14], and to Constantinescu and Cornea in their study of Brelot harmonic spaces in 1965 [9].

$f \in C^{\infty}(M, N)$ is a harmonic morphism if for every harmonic function $\phi$ on an open set $A \subseteq N$ such that $\phi^{-1}(A)$ is non-empty, the composition $f \circ \phi$ is harmonic on $\phi^{-1}(A) \subseteq M$.

A sufficient and necessary condition for a smooth map to be a harmonic morphism is obtained by Fuglede [10] and Ishihara [13]. It asserts that $f$ is a harmonic morphism if and only if $f$ is harmonic and is horizontally weakly conformal; see Section 3 . This result gives a local characterization of harmonic morphisms. In contrast, the stability of harmonic mappings is a global condition. A connection between these two is established in Section 3 in this paper, via an algebraic equation which stable harmonic maps into certain positively curved spaces satisfy. In fact, we show that any smooth stable harmonic map from any compact smooth Riemannian manifold into the 2-sphere $S^{2}$ with the standard Euclidean metric is a harmonic morphism.

Now we summarize the main results in this paper. In Section 2 we obtain a rank estimate which asserts that the rank of the differential of a smooth stable harmonic map from a compact manifold into $\mathbb{C} P^{k}$ with the Fubini-Study metric has to be even; see Theorem 2.4. Further, in Theorem 2.1 we prove that the map is pair-wise conformal (see Theorem 2.1 and Corollary 2.3). In Section 3, we show that any smooth stable harmonic map into $S^{2}$ is a harmonic morphism. Section 4 focuses on the case where the domain is a compact 3-manifold. Frist, in Theorem 4.1 we prove that the image set is an algebraic curve in $\mathbb{C} P^{k}$ with its singular locus consisting of only finitely many points at worst. Then in Theorem 4.6, we show that the stable harmonic map is a harmonic morphism away from the preimage of the singular locus to the algebraic curve substracting its singular locus. In Section 5 , by composing the stable harmonic map with a standard projection in $\mathbb{C} P^{k}$, we obtain a non-constant harmonic morphism from the entire domain into $\mathbb{C} P^{1}$. Hence, we find a topological obstruction on the existence of stable harmonic map from $M^{3}$ into $\mathbb{C} P^{k}$, namely, $M^{3}$ is necessarily a Seifert fibre space; see Theorem 5.1.

The author would like to thank P. Li, R. Schoen, L.F. Tam and A. Treibergs for their valuable comments on this work and for constant 
encouragement, and also to thank F. Burstall and J.C. Wood for their interest in this work and suggestive communication. The author is also grateful to the referee for his suggestions that led to some improvements in the presentation of the paper.

\section{Rank estimates}

Let $f$ be a smooth mapping from a compact Riemannian manifold $M$ without boundary into the complex projective space $\mathbb{C} P^{k}$ with the Fubini-Sduty metric. We denote the Riemannian matric on $M$ and the Fubini-Study metric on $\mathbb{C} P^{k}$ by $g$ and $h$ respectively. For any point $p \in M$, let $\left\{e_{1}, \ldots, e_{n}\right\}$ be an orthonormal basis for $T_{p} M$, the tangent space of $M$ at $p$, where $n=\operatorname{dim} M$. Let $\left(z^{1}, \ldots, z^{k}\right)$ be the complex coordinates in a neighborhood of $f(p)$ in $\mathbb{C} P^{k}$, and write $z^{\alpha}=x^{\alpha}+i y^{\alpha}$ for $\alpha=1, \ldots, k=\operatorname{dim}_{C} \mathbb{C} P^{k}$. For simplicity, we introduce the following convention throughout the paper:

$$
\partial_{\alpha}=\frac{\partial}{\partial x^{\alpha}}, \quad \partial_{\tilde{\alpha}}=\frac{\partial}{\partial y^{\alpha}} .
$$

Also we denote the complex structure on $\mathbb{C} P^{k}$ by $J$. It is well known that $J$ can be regarded as a real linear map from $T_{f(p)} \mathbb{C} P^{k}$ to $T_{f(p)} \mathbb{C} P^{k}$ such that

$$
J \partial_{\alpha}=\partial_{\tilde{\alpha}}, J \partial_{\tilde{\alpha}}=-\partial_{\alpha} .
$$

In the following, we set, for the Riemannian curvature tensor,

$$
R(X, Y, Z, W)=\langle R(X, Y) Z, W\rangle .
$$

It is clear that

$$
\begin{gathered}
R(X, Y, Z, W)=R(Z, W, X, Y) \\
R(X, Y, Z, W)=-R(Y, X, Z, W)=-R(X, Y, W, Z) .
\end{gathered}
$$

Notice that the above convention is different by a minus sign from [15]. We also write the differential in local coordinates as follows:

$$
d f\left(e_{i}\right)=f_{i}^{\alpha} \partial_{\alpha}+f_{i}^{\tilde{\alpha}} \partial_{\tilde{\alpha}},
$$

where the summation convention is used. We have 
Theorem 2.1. Let $(M, g)$ be a compact n-dimensional Riemannian manifold without boundary. If $f$ is a smooth stable harmonic map from $M$ to $\mathbb{C} P^{k}$ with the Fubini-Study metric $h$, then for any $\alpha, \beta=1, \ldots, k=$ $\operatorname{dim}_{C} \mathbb{C} P^{k}$

$$
\begin{aligned}
& \sum_{i=1}^{n} f_{i}^{\alpha} f_{i}^{\beta}=\sum_{i=1}^{n} f_{i}^{\tilde{\alpha}} f_{i}^{\tilde{\beta}} \\
& \sum_{i=1}^{n} f_{i}^{\alpha} f_{i}^{\tilde{\beta}}=-\sum_{i=1}^{n} f_{i}^{\tilde{\alpha}} f_{i}^{\beta} .
\end{aligned}
$$

Proof. Since $\mathbb{C} P^{k}$ has constant holomorphic sectional curvature $c$, Proposition 7.3 in [15] asserts that for any $X, Y, Z, W \in T_{f(p)} \mathbb{C} P^{k}$

$$
\begin{aligned}
-R(X, Y, Z, W)=\frac{c}{4}(h & (X, Z) h(Y, W)-h(X, W) h(Y, Z) \\
& +h(X, J Z) h(Y, J W)-h(X, J W) h(Y, J Z) \\
& +2 h(X, J Y) h(Z, J W)) .
\end{aligned}
$$

Therefore

$$
\begin{aligned}
&-\frac{4}{c} \sum_{i=1}^{n} \quad\left\langle R\left(d f\left(e_{i}\right), X\right) J d f\left(e_{i}\right), Y\right\rangle \\
&=\sum_{i=1}^{n}\left(h\left(d f\left(e_{i}\right), J d f\left(e_{i}\right)\right) h(X, Y)-h\left(d f\left(e_{i}\right), Y\right) h\left(X, J d f\left(e_{i}\right)\right)\right. \\
& \quad+h\left(d f\left(e_{i}\right), J^{2} d f\left(e_{i}\right)\right) h(X, J Y) \\
& \quad-h\left(d f\left(e_{i}\right), J Y\right) h\left(X, J^{2} d f\left(e_{i}\right)\right) \\
&\left.\quad+2 h\left(d f\left(e_{i}\right), J X\right) h\left(J d f\left(e_{i}\right), J Y\right)\right) \\
&=\sum_{i=1}^{n}\left(h\left(d f\left(e_{i}\right), J d f\left(e_{i}\right)\right) h(X, Y)+h\left(d f\left(e_{i}\right), Y\right) h\left(J X, d f\left(e_{i}\right)\right)\right. \\
& \quad-h\left(d f\left(e_{i}\right), d f\left(e_{i}\right)\right) h(X, J Y)+h\left(d f\left(e_{i}\right), J Y\right) h\left(X, d f\left(e_{i}\right)\right) \\
&\left.\quad+2 h\left(d f\left(e_{i}\right), J X\right) h\left(d f\left(e_{i}\right), Y\right)\right) \\
&=\sum_{i=1}^{n}\left(h\left(d f\left(e_{i}\right), J d f\left(e_{i}\right)\right) h(X, Y)+3 h\left(d f\left(e_{i}\right), Y\right) h\left(J X, d f\left(e_{i}\right)\right)\right. \\
&\left.\quad-h\left(d f\left(e_{i}\right), d f\left(e_{i}\right)\right) h(X, J Y)+h\left(d f\left(e_{i}\right), J Y\right) h\left(X, d f\left(e_{i}\right)\right)\right),
\end{aligned}
$$


where we have used the fact that $h$ is Hermitian, i.e., $h(J Z, J W)=$ $h(Z, W)$ for any $Z, W \in T_{f(p)} \mathbb{C} P^{k}$. Similarly

$$
\begin{aligned}
&-\frac{4}{c} \sum_{i=1}^{n} \quad\left\langle R\left(d f\left(e_{i}\right), J X\right) d f\left(e_{i}\right), Y\right\rangle \\
&=\sum_{i=1}^{n}( h\left(d f\left(e_{i}\right), d f\left(e_{i}\right)\right) h(J X, Y)-h\left(d f\left(e_{i}\right), Y\right) h\left(J X, d f\left(e_{i}\right)\right) \\
&+h\left(d f\left(e_{i}\right), J d f\left(e_{i}\right)\right) h(J X, J Y) \\
&-h\left(d f\left(e_{i}\right), J Y\right) h\left(J X, J d f\left(e_{i}\right)\right) \\
&\left.+2 h\left(d f\left(e_{i}\right), J^{2} X\right) h\left(d f\left(e_{i}\right), J Y\right)\right) \\
&(3) \quad \\
&=\sum_{i=1}^{n} \quad-h\left(d f\left(e_{i}\right), d f\left(e_{i}\right)\right) h(X, J Y)-h\left(d f\left(e_{i}\right), Y\right) h\left(J X, d f\left(e_{i}\right)\right) \\
&+h\left(d f\left(e_{i}\right), J d f\left(e_{i}\right)\right) h(X, Y)-h\left(d f\left(e_{i}\right), J Y\right) h\left(X, d f\left(e_{i}\right)\right) \\
&\left.-2 h\left(d f\left(e_{i}\right), X\right) h\left(d f\left(e_{i}\right), J Y\right)\right) \\
&=\sum_{i=1}^{n}(-h\left(d f\left(e_{i}\right), d f\left(e_{i}\right)\right) h(X, J Y)-h\left(d f\left(e_{i}\right), Y\right) h\left(J X, d f\left(e_{i}\right)\right) \\
&+h\left(d f\left(e_{i}\right), J d f\left(e_{i}\right)\right) h(X, Y) \\
&\left.-3 h\left(d f\left(e_{i}\right), J Y\right) h\left(X, d f\left(e_{i}\right)\right)\right) .
\end{aligned}
$$

Now by subtracting (3) from (2), we obtain

$$
\begin{gathered}
\frac{4}{c} \sum_{i=1}^{n}\left\langle R\left(d f\left(e_{i}\right), X\right) J d f\left(e_{i}\right), Y\right\rangle-\frac{4}{c} \sum_{i=1}^{n}\left\langle R\left(d f\left(e_{i}\right), J X\right) d f\left(e_{i}\right), Y\right\rangle \\
=\sum_{i=1}^{n}\left(4 h\left(d f\left(e_{i}\right), Y\right) h\left(J X, d f\left(e_{i}\right)\right)\right. \\
\left.+4 h\left(d f\left(e_{i}\right), J Y\right) h\left(X, d f\left(e_{i}\right)\right)\right) .
\end{gathered}
$$

Recall that in [19] and [5], the following proposition is derived by an argument introduced by Lawson and Simons in [16] on the second variation of energy via holomorphic vector fields.

Proposition 2.2. Suppose that $M$ is a smooth compact Riemannian manifold and $N$ is a compact irreducible Hermitian symmetric space with a complex structure $J$. Let $f: M \rightarrow N$ be a stable harmonic map. 
Then for any $p \in M$ and $X \in T_{f(p)} N$, we have

(5) $\quad \sum_{i=1}^{n}\left(J R^{N}\left(d f\left(e_{i}\right), X\right) d f\left(e_{i}\right)-R^{N}\left(d f\left(e_{i}\right), J X\right) d f\left(e_{i}\right)\right)=0$,

where $n=\operatorname{dim} M,\left\{e_{1}, \ldots, e_{n}\right\}$ is an orthonormal basis for $T_{p} M$, and $R^{N}$ denotes the curvature tensor of $N$.

Since $f$ is a stable harmonic map into $\mathbb{C} P^{k}$ which is irreducible Hermitian symmetric of compact type, by combining (5) with the fact that the curvature $R$ commutes with $J$ for a Kähler manifold (see Proposition 4.5 in [15]) i.e., for all vector fields $W, Z$

$$
R(W, Z) J=J R(W, Z)
$$

we can obtain

(6) $\quad \sum_{i=1}^{n}\left\langle R\left(d f\left(e_{i}\right), X\right) J d f\left(e_{i}\right), Y\right\rangle=\sum_{i=1}^{n}\left\langle R\left(d f\left(e_{i}\right), J X\right) d f\left(e_{i}\right), Y\right\rangle$.

Then from (6), the left-hand side of (4) is 0 . Thereby, the right-hand side of (4) has to be 0 , so we have

(7) $\sum_{i=1}^{n}\left(h\left(d f\left(e_{i}\right), J X\right) h\left(d f\left(e_{i}\right), Y\right)+h\left(d f\left(e_{i}\right), X\right) h\left(d f\left(e_{i}\right), J Y\right)\right)=0$.

Next, we pick some special $X$ and $Y$ for (7). In order to see what $X$ and $Y$ to choose, we first work out a general formula in local coordinates. Let

$$
X=a^{\alpha} \partial_{\alpha}+b^{\tilde{\alpha}} \partial_{\tilde{\alpha}}, Y=c^{\alpha} \partial_{\alpha}+d^{\tilde{\alpha}} \partial_{\tilde{\alpha}}
$$

be arbitrary tangent vectors in $T_{f(p)} \mathbb{C} P^{k}$, where $a^{\alpha}, b^{\tilde{\alpha}}, c^{\alpha}, d^{\tilde{\alpha}} \in \mathrm{R}$ are arbitrary constants for $\alpha, \tilde{\alpha}=1, \ldots, k$. We notice that in the complex local coordinates around $f(p)$ on $\mathbb{C} P^{k}$

$$
\begin{aligned}
d f\left(e_{i}\right) & =f_{i}^{\alpha} \partial_{\alpha}+f_{i}^{\tilde{\alpha}} \partial_{\tilde{\alpha}}, \\
J d f\left(e_{i}\right) & =-f_{i}^{\tilde{\alpha}} \partial_{\alpha}+f_{i}^{\alpha} \partial_{\tilde{\alpha}}, \\
J X & =-b^{\tilde{\alpha}} \partial_{\alpha}+a^{\alpha} \partial_{\tilde{\alpha}} \\
J Y & =-d^{\tilde{\alpha}} \partial_{\alpha}+c^{\alpha} \partial_{\tilde{\alpha}} .
\end{aligned}
$$


Hence, we have from (7) that

$$
\begin{aligned}
& 0=\sum_{i=1}^{n}\left(h\left(f_{i}^{\alpha} \partial_{\alpha}+f_{i}^{\tilde{\alpha}} \partial_{\tilde{\alpha}}, c^{\beta} \partial_{\beta}+d^{\tilde{\beta}} \partial_{\tilde{\beta}}\right) h\left(f_{i}^{\gamma} \partial_{\gamma}+f_{i}^{\tilde{\gamma}} \partial_{\tilde{\gamma}},-b^{\tilde{\delta}} \partial_{\delta}+a^{\delta} \partial_{\tilde{\delta}}\right)\right. \\
& \left.+h\left(f_{i}^{\alpha} \partial_{\alpha}+f_{i}^{\tilde{\alpha}} \partial_{\tilde{\alpha}}, a^{\beta} \partial_{\beta}+b^{\bar{\beta}} \partial_{\tilde{\beta}}\right) h\left(f_{i}^{\gamma} \partial_{\gamma}+f_{i}^{\tilde{\gamma}} \partial_{\tilde{\gamma}},-d^{\tilde{\delta}} \partial_{\delta}+c^{\delta} \partial_{\tilde{\delta}}\right)\right) \\
& =\sum_{\alpha, \beta}^{k} \sum_{i=1}^{n}\left(\left(f_{i}^{\alpha} c^{\alpha}+f_{i}^{\tilde{\alpha}} d^{\tilde{\alpha}}\right)\left(-f_{i}^{\beta} b^{\tilde{\beta}}+f_{i}^{\tilde{\beta}} a^{\beta}\right)\right. \\
& \left.+\left(f_{i}^{\alpha} a^{\alpha}+f_{i}^{\tilde{\alpha}} b^{\tilde{\alpha}}\right)\left(-f_{i}^{\beta} d^{\tilde{\beta}}+f_{i}^{\bar{\beta}} c^{\beta}\right)\right) \\
& =\sum_{\alpha, \beta}^{k} \sum_{i=1}^{n}\left(-\left(a^{\alpha} d^{\bar{\beta}}+c^{\alpha} b^{\bar{\beta}}\right) f_{i}^{\alpha} f_{i}^{\beta}+\left(a^{\beta} d^{\bar{\alpha}}+b^{\bar{\alpha}} c^{\beta}\right) f_{i}^{\bar{\alpha}} f_{i}^{\tilde{\beta}}\right. \\
& \left.+\left(a^{\beta} c^{\alpha}+a^{\alpha} c^{\beta}\right) f_{i}^{\alpha} f_{i}^{\tilde{\beta}}-\left(b^{\tilde{\beta}} d^{\tilde{\alpha}}+b^{\tilde{\alpha}} d^{\tilde{\beta}}\right) f_{i}^{\tilde{\alpha}} f_{i}^{\beta}\right) .
\end{aligned}
$$

(8)

Now we just need to choose some special values for $a^{\alpha}, b^{\tilde{\alpha}}, c^{\alpha}, d^{\tilde{\alpha}}$. First, let $\left(b^{\tilde{1}}, \ldots, b^{\tilde{k}}\right)=\left(d^{\tilde{1}}, \ldots, d^{\tilde{k}}\right)=(0, \ldots, 0)$ in $(8)$. Then

$$
\sum_{\alpha, \beta}^{k} \sum_{i=1}^{n}\left(a^{\beta} c^{\alpha}+a^{\alpha} c^{\beta}\right) f_{i}^{\alpha} f_{i}^{\tilde{\beta}}=0 .
$$

Setting $\left(a^{1}, \ldots, a^{k}\right)=(0, \ldots, 1,0, \ldots, 0)$ where 1 is in the $\gamma$ th place and $\left(c^{1}, \ldots, c^{k}\right)=(0, \ldots, 1,0, \ldots, 0)$ where 1 is in the $\delta$ th place, we obtain

$$
\sum_{i=1}^{n}\left(f_{i}^{\gamma} f_{i}^{\tilde{\delta}}+f_{i}^{\delta} f_{i}^{\tilde{\gamma}}\right)=0
$$

Second, let $\left(b^{\tilde{1}}, \ldots, b^{\tilde{k}}\right)=\left(c^{1}, \ldots, c^{k}\right)=(0, \ldots, 0)$. Then

$$
\sum_{\alpha, \beta}^{k} \sum_{i=1}^{n} a^{\alpha} d^{\tilde{\beta}} f_{i}^{\alpha} f_{i}^{\beta}=\sum_{i=1}^{n} a^{\beta} d^{\tilde{\alpha}} f_{i}^{\tilde{\alpha}} f_{i}^{\bar{\beta}}
$$

Setting $\left(a^{1}, \ldots, a^{k}\right)=(0, \ldots, 1,0, \ldots, 0)$ where 1 is in the $\gamma$ th place and $\left(d^{\tilde{1}}, \ldots, d^{\bar{k}}\right)=(0, \ldots, 1,0, \ldots, 0)$ where 1 is in the $\tilde{\delta}$ th place, we get

$$
\sum_{i=1}^{n} f_{i}^{\gamma} f_{i}^{\delta}=\sum_{i=1}^{n} f_{i}^{\bar{\gamma}} f_{i}^{\bar{\delta}}
$$

This completes the proof of Theorem 2.1 . 
In particular, if we let $\gamma=\delta$ in (9) and (10), we have

Corollary 2.3. Let $(M, g)$ be a compact Riemannian manifold. If $f$ is a smooth stable harmonic map from $M$ to $\mathbb{C} P^{k}$ with the Fubini-Study metric $h$, then for any $\gamma=1, \ldots, m=\operatorname{dim}_{C} N$

$$
\begin{aligned}
& \sum_{i=1}^{n} f_{i}^{\gamma} f_{i}^{\tilde{\gamma}}=0 \\
& \sum_{i=1}^{n} f_{i}^{\gamma} f_{i}^{\gamma}=\sum_{i=1}^{n} f_{i}^{\tilde{\gamma}} f_{i}^{\tilde{\gamma}} .
\end{aligned}
$$

An interesting application of Theorem 2.1 and Corollary 2.3 is that they allow us to draw a conclusion on the rank of the differential of the stable harmonic map. In fact, we have

Theorem 2.4. Let $(M, g)$ be a compact n-dimensional Riemannian manifold without boundary. If $f$ is a smooth stable harmonic map from $M$ into $\mathbb{C} P^{k}$ with the Fubini-Study metric, then the rank of the differential of $f$ is even at any point in $M$.

Proof. We use the same notation as before. At any point $p \in M$, we set $a_{i}=\left(f_{1}^{i}, \ldots, f_{n}^{i}\right)$ and $b_{i}=\left(f_{1}^{\bar{i}}, \ldots, f_{n}^{\bar{i}}\right)$ for $i=1, \ldots, k$. So we can represent the differential in terms of matrix as follows:

$$
d f_{p}=\left(\begin{array}{ccc}
f_{1}^{1} \ldots & f_{n}^{1} \\
\ldots \\
f_{1}^{k} \ldots f_{n}^{k} \\
f_{1}^{\tilde{1}} \ldots f_{n}^{\tilde{1}} \\
\ldots \\
f_{1}^{\tilde{k}} \ldots f_{n}^{\tilde{k}}
\end{array}\right)_{2 k \times n}=\left(\begin{array}{c}
a_{1} \\
\ldots \\
a_{k} \\
b_{1} \\
\ldots \\
b_{k}
\end{array}\right) .
$$

If the rank of $d f_{p}$ is 0 , the theorem is proved at the point $p$. So we assume that the rank of $d f_{p}$ is not 0 . Without loss of generality, let $a_{1} \neq(0, \ldots, 0)$. By Corollary 2.3 , we have that

$$
\begin{aligned}
& a_{1} \cdot b_{1}=0, \\
& a_{1} \cdot a_{1}=b_{1} \cdot b_{1} \neq 0 .
\end{aligned}
$$

Thus $a_{1}$ and $b_{1}$ are perpendicular to each other and have the same length $\lambda=\left|a_{1}\right|=\left|b_{1}\right|$. Hence, there is an orthonormal linear map $\rho$ in 
$T_{f(p)} \mathbb{C} P^{k}$ such that in the fixed orthonormal basis $\left\{\partial_{1}, \partial_{\overline{1}}, \ldots, \partial_{k}, \partial_{\tilde{k}}\right\}$

$$
\begin{aligned}
& \rho\left(a_{1}\right)=(\lambda, 0,0, \ldots, 0), \\
& \rho\left(b_{1}\right)=(0, \lambda, 0, \ldots, 0) .
\end{aligned}
$$

Furthermore, $\rho$ preserves the inner product in $T_{p} M$. Hence, Theorem 2.1 , for any $i, j=1, \ldots, k$, yields

$$
\begin{aligned}
& \rho\left(a_{i}\right) \cdot \rho\left(a_{j}\right)=\rho\left(b_{i}\right) \cdot \rho\left(b_{j}\right), \\
& \rho\left(a_{i}\right) \cdot \rho\left(b_{j}\right)=-\rho\left(a_{j}\right) \cdot \rho\left(b_{i}\right) .
\end{aligned}
$$

For any $i=1, \ldots, k$, denote

$$
\begin{aligned}
& \rho\left(a_{i}\right)=c_{i}=\left(c_{1}^{i}, \ldots, c_{n}^{i}\right) \\
& \rho\left(b_{i}\right)=d_{i}=\left(d_{1}^{i}, \ldots, d_{n}^{i}\right) .
\end{aligned}
$$

In particular, for any $i=1, \ldots, k$, we have

$$
\begin{aligned}
& \lambda c_{1}^{i}=\rho\left(a_{i}\right) \cdot \rho\left(a_{1}\right)=\rho\left(b_{i}\right) \cdot \rho\left(b_{1}\right)=\lambda d_{2}^{i} \\
& \lambda c_{2}^{i}=\rho\left(a_{i}\right) \cdot \rho\left(b_{1}\right)=-\rho\left(b_{i}\right) \cdot \rho\left(a_{1}\right)=-\lambda d_{1}^{i} .
\end{aligned}
$$

Thus $\lambda \neq 0$ implies that

$$
c_{1}^{i}=d_{2}^{i}, \quad c_{2}^{i}=-d_{1}^{i}
$$

Now we consider the following submatrix of $\rho\left(d f_{p}\right)$ :

$$
\Pi=\left(\begin{array}{c}
\pi_{1} \\
\ldots \\
\pi_{k-1} \\
\tilde{\pi}_{1} \\
\ldots \\
\tilde{\pi}_{k-1}
\end{array}\right)=\left(\begin{array}{ccc}
c_{3}^{2} & \ldots & c_{n}^{2} \\
\ldots \\
c_{3}^{k} & \ldots & c_{n}^{k} \\
d_{3}^{2} & \ldots & d_{n}^{2} \\
\ldots & \\
d_{3}^{k} & \ldots & d_{n}^{k}
\end{array}\right)_{2(k-1) \times(n-2)}
$$

which is obtained from $\rho\left(d f_{p}\right)$ by crossing out the first row $c_{1}$, the $(k+1)$ st row $d_{1}$ and the first two columns. We claim that the matrix 
$\Pi$ still has the same two properties as $\rho\left(d f_{p}\right)$ has; namely,

$$
\begin{aligned}
\pi_{i} \cdot \pi_{j} & =\sum_{s=3}^{n} c_{s}^{i} c_{s}^{j} \\
& =\sum_{s=1}^{n} c_{s}^{i} c_{s}^{j}-c_{1}^{i} c_{1}^{j}-c_{2}^{i} c_{2}^{j} \\
& =\sum_{s=1}^{n} d_{s}^{i} d_{s}^{j}-c_{1}^{i} c_{1}^{j}-c_{2}^{i} c_{2}^{j} \\
& =\sum_{s=1}^{n} d_{s}^{i} d_{s}^{j}-d_{2}^{i} d_{2}^{j}-\left(-d_{1}^{i}\right)\left(-d_{1}^{j}\right) \\
& =\sum_{s=3}^{n} d_{s}^{i} d_{s}^{j} \\
& =\tilde{\pi}_{i} \cdot \tilde{\pi}_{j},
\end{aligned}
$$

and

$$
\begin{aligned}
\pi_{i} \cdot \tilde{\pi}_{j} & =\sum_{s=3}^{n} c_{s}^{i} d_{s}^{j} \\
& =\sum_{s=1}^{n} c_{s}^{i} d_{s}^{j}-c_{1}^{i} d_{1}^{j}-c_{2}^{i} d_{2}^{j} \\
& =-\sum_{s=1}^{n} d_{s}^{i} c_{s}^{j}-c_{1}^{i} d_{1}^{j}-c_{2}^{i} d_{2}^{j} \\
& =-\sum_{s=1}^{n} d_{s}^{i} c_{s}^{j}-d_{2}^{i}\left(-c_{2}^{j}\right)-\left(-d_{1}^{i}\right) c_{1}^{j} \\
& =-\sum_{s=3}^{n} d_{s}^{i} c_{s}^{j} \\
& =-\tilde{\pi}_{i} \cdot \pi_{j},
\end{aligned}
$$

From the row reduction of $\rho\left(d f_{p}\right)$ it is easy to see that

$$
\operatorname{rank} d f_{p}=\operatorname{rank} \rho\left(d f_{p}\right)=2+\operatorname{rank} \Pi .
$$

Since $\Pi$ enjoys the two properties, we can do the same discussion on $\Pi$ as we just did on $\rho\left(d f_{p}\right)$. Continuing this procedure (so we have (14) and (15) at each step), we see that each time we obtain 2 on 
the right hand of (16) and a smaller matrix with the two properties. Finally, when the process stops, the remaining submatrix, if it exists, has to be identically 0 , due to the fact that this matrix still has the two properties, namely, its rows are pair-wise perpendicular and with the same length in the sense of Corollary 2.3. Hence the proof of Theorem 2.4 is completed.

\section{Stable harmonic maps into $S^{2}$}

The main result in this section is given by

Theorem 3.1. Let $f$ be a stable harmonic map from any compact smooth Riemannian manifold $(M, g)$ into $S^{2}$ with the standard metric. Then $f$ is a harmonic morphism.

Before giving the proof of Theorem 3.1, let us recall some facts about harmonic morphisms.

Definition 3.2. A smooth map $\phi:(M, g) \rightarrow(N, h)$ is horizontally weakly conformal if at each point $p$ in $M$

$$
g^{i j} \frac{\partial \phi^{\alpha}}{\partial x^{i}} \frac{\partial \phi^{b}}{\partial x^{j}}=\lambda(p) h^{\alpha \beta},
$$

where $\lambda$ is a non-negative continuous function.

The following result belongs to B. Fuglede [10] and T. Ishihara [13].

Proposition 3.3. A smooth map between two Riemannian manifolds is a harmonic morphism if and only if it is both harmonic and horizontally weakly conformal.

Proof. (Theorem 3.1) It is immediate by Definition 3.2 that (11) and (12) in Corollary 2.3 together imply that $f$ is horizontally weakly conformal. Therefore by Fuglede and Ishihara's result (Proposition 3.3 ), we conclude that $f$ is a harmonic morphism. This completes the proof of Theorem 3.1.

Remark 1. The converse of Theorem 3.1 is not true. In fact, the Hopf fibration from $S^{3}$ to $S^{2}$ is a harmonic morphism but not stable.

Since stable harmonic map into $S^{2}$ is a harmonic morphism, it enjoys all the properties that the latter has. We collect some interesting facts in

Corollary 3.4. Let $f$ be a non-constant stable harmonic map from any compact manifold into $S^{2}$ with the standard metric. Then the following hold: 
(a) $f$ is an open mapping and hence surjective.

(b) The set $\mathcal{S}$ of all critical points of $f$ cannot disconnect any open ball in $M$. Furthermore, if $M$ is real analytic, then $\mathcal{S}$ is a real analytic variety of codimension $\geq 2$.

(c) The fibres over regular points are minimal submanifolds of $M$.

(d) $\operatorname{rank}\left(d f_{p}\right)=2$ or 0 at every point $p \in M$.

Proof. After knowing that $f$ is a harmonic morphism, (a) and (b) are due to B. Fuglede [10], (c) is due to P. Baird and J. Eells [2]. (d) follows from $f$ being horizontally weakly conformal.

In general, it is very interesting to know whether one can find an energy minimizer in a given homotopy class if the target manifolds do not have non-positive sectional curvature. P.F. Leung [17] proves that any stable harmonic map from compact manifold into $S^{n}$ for $n \geq 3$ is constant. Therefore, if the target space is $S^{3}$, then one cannot find smooth energy minimizers in any non-trivial homotopy class. However, this is not true in general if the target is $S^{2}$, since Lichnerowicz [18] observed that any (anti) holomorphic maps between two Kähler manifolds minimize the energy in their homotopy classes. Nevertheless, a consequence of Theorem 3.1 provides a necessary condition on stable harmonic maps into $S^{2}$. In particular, we have

Corollary 3.5. Let $M$ be any compact 3-manifold which is not a Seifert fibre space. Then any stable harmonic map from $M$ into $S^{2}$ is constant.

Proof. Theorem 3.1 implies that the stable harmonic map $f$ is a harmonic morphism. If $f$ is not constant, a result of P. Baird and J.C. Wood [3] asserts that the foliation given by the fibres of $f$ gives $M$ the structure of a $C^{\infty}$ Seifert fibre space. This contradicts the assumption on $M$.

We should point out here that closed 3-dimensional Seifert fibre spaces are completely classified by the following result due to $\mathrm{W}$. Thurston [22].

Proposition 3.6. Any closed Seifert fibre space $(M, \mathcal{F})$ of dimension three is homeomorphic to $E / \Gamma$, where $\Gamma$ is a group of isometries of $E$ acting freely, properly and discontinuously on $E$, and $E$ is one of the following Riemannian manifolds: (1) $R^{2} \times R$, (2) $S^{2} \times R$, (3) $H^{2} \times R$, (4) $S^{3}$, the metric on each of these is the product of standard metrics; (5) $R^{3}$ with the metric $d s^{2}=d x^{2}+d y^{2}+(d z-x d y)^{2}$; (6) 
$R_{+}^{3}=\left\{(x, y, z) \in R^{3}: y>0\right\}$ with the metric $d s^{2}=\left(d x^{2}+d y^{2}\right) / y^{2}+$ $(d x / y+d z)^{2} ;(7) Q_{p, q}=\left\{\left(z_{1}, z_{2}\right) \in \mathbb{C}^{2}:\left|z_{1}\right|^{2} / p^{2}+\left|z_{2}\right|^{2} / q^{2}=1\right\}$ with the induced metric from the standard metric on $R^{4}$. The foliation $\mathcal{F}$ is obtained from the standard foliation on $E$.

\section{Maps from compact 3-manifolds}

As we have seen in the last section, there is a strong restriction on the rank of the differential of a smooth stable harmonic map into $\mathbb{C} P^{k}$. Specially, if the dimension of the domain manifold is odd and not larger than $2 k$, the real dimension of $\mathbb{C} P^{k}$, then the differential cannot have full rank at any point. In this section, we shall discuss the stable harmonic mappings from compact 3-manifolds into $\mathbb{C} P^{k}$.

First, let us recall that an analytic subvariety $V$ of a complex manifold is a subset given locally as the common zeros of a finite collection of holomorphic functions. A point $p \in V$ is called a smooth point of $V$ if $V$ is a submanifold near $p$, otherwise it is called a singular point. The singular locus $\mathcal{S}$ of $V$ is the set of all singular ponits of $V$. An algebraic subvariety of $\mathbb{C P}$ is the subset expressed locally as common zeros of finitely many homogeneous polynomials. The important theorem of Chow asserts that any analytic subvariety of the complex projective space is algebraic; see for example [11]. Finally, a one-dimensional algebraic subvariety of $\mathbb{C} P^{k}$ is called an algebraic curve in $\mathbb{C} P^{k}$.

The first main result in this section is the following assertion on the image set.

Theorem 4.1. Let $M$ be a smooth compact manifold of dimension three without boundary. Let $f$ be a non-constant stable harmonic map from $M$ into $\mathbb{C} P^{k}$ with the Fubini-Study metric. Then the image set $f(M)$ of $M$ under $f$ is a compact algebraic curve in $\mathbb{C P}^{k}$ and the singular locus $\mathcal{S}$ of $f(M)$ consists of finitely many points at most.

The proof of Theorem 4.1 follows from the following sequence of lemmas. The lemma below tells us that away from a small set $f(M)$ is a smooth surface due to the rank restriction.

Lemma 4.2. Let $M$ be a smooth compact manifold of dimension three without boundary. Let $f$ be a non-constant smooth stable harmonic map from $M$ into $\mathbb{C P}^{k}$ with the Fubini-Study metric. Then the set $f(M)-f(K)-\bigcup_{i} q_{i}$ is a smooth surface in $\mathbb{C} P^{k}$, where $K=\{x \in M$ : 
$\left.d f_{x}=0\right\}$ and $\bigcup_{i} q_{i}$ is the union of at most countably many points.

Proof. First, by Theorem 2.2, we know that for any point $p \in M$, the rank of $d f_{p}$ is either 0 or 2 since $\operatorname{dim} M=3$. Let $p \in M$ be any rank-2 point. Then $d f_{p} \neq 0$, and there is an open neighborhood $U$ of $p$ in $M$ such that rank $d f_{q} \neq 0$, hence rank $d f_{q}=2$, for any point $q \in U$. The tangent space of $M$ at $p$ is decomposed orthogonally as follows $T_{p} M=\operatorname{Ker}\left(d f_{p}\right) \oplus H_{p}$ where $H_{p}=\left(\operatorname{Ker}\left(d f_{p}\right)\right)^{\perp}$. Let $e_{1}$ be a unit vector in $\operatorname{Ker}\left(d f_{p}\right)$, and $\left\{e_{2}, e_{3}\right\}$ be an orthonormal basis for $H_{p}$. Next, consider a small open neighborhood $H$ of 0 in $H_{p}$ such that the exponential map $\exp _{p}$ is diffeomorphic on $H$. Define $F: H \rightarrow \mathbb{C} P^{k}$ by $F=f \circ \exp _{p}$. It is clear that $\operatorname{rank} d F_{(x, y)}=2$ for any $(x, y) \in H$. The inverse function theorem then asserts that $H$ is diffeomorphic to $F(H) \subset \mathbb{C} P^{k}$. Thus $F(H)$ is a smooth surface in $\mathbb{C} P^{k}$. But $F(H)=f\left(\exp _{p}(H)\right)$, hence $f\left(\exp _{p}(H)\right)$ is a smooth surface in $\mathbb{C} P^{k}$. Moreover, since $\exp _{p}$ is a diffeomorphism, $\exp _{p}(H)$ is a smooth surface in $M$.

Second, we construct an open neighborhood $V_{p}$ of $p$ in $M$ such that $f\left(V_{p}\right)=f\left(\exp _{\rho}(H)\right)$ via the fibres over $\exp _{p}(H)$. In fact, for any point $q \in f\left(\exp _{p}(H)\right)$, it is a regular value of $f:$ the preimage of $f\left(\exp _{p}(H)\right)$ $\rightarrow f\left(\exp _{p}(H)\right)$. According to the regular value theorem, the preimage $f^{-1}(q)$ is a smooth curve in $M$ with no end points. So $f^{-1}(q)$ can be viewed as a fibre over the surface $\exp _{p}(H)$ and it crosses the surface. Therefore we can find an open neighborhood $V_{p}$ of $p$ in $M$, which contains at least an open piece of $f\left(\exp _{p}(H)\right)$. From the construction of $V_{p}$, it is easy to see that $f\left(V_{p}\right)=f\left(\exp _{p}(H)\right)$ is a smooth surface in $\mathbb{C} P^{k}$. In fact, by Lemma 4.3 , which we are going to show below, $f\left(V_{p}\right)$ is a 1-dimensional complex submanifold of $\mathbb{C} P^{k}$.

Finally, let $V_{p_{1}}$ and $V_{p_{2}}$ be any two open neighborhoods, which are constructed as before around points $p_{1}$ and $p_{2}$ respectively. Set $T=$ $\left\{x \in f\left(V_{p_{1}}\right) \cap f\left(V_{p_{2}}\right): f\left(V_{p_{1}}\right) \cup f\left(V_{p_{2}}\right)\right.$ fails to be a smooth surface at $\left.x\right\}$. If $T$ is nonempty, then $T$ consists of isolated points, since both $f\left(V_{p_{1}}\right)$ and $f\left(V_{p_{2}}\right)$ are 1-dimensional complex submanifolds of $\mathbb{C} P^{k}$ (Lemma 4.3) and the theory of complex one variable asserts that if any two holomorphic functions equal on a set which contains a limit point, the two functions are identicaly same. So $T$ consists of countably many points at most. Since $M-K$ can be covered by a countable family of $V_{p}$, the union of the sets $T$ consists of at most a countable family of points $\left\{q_{i}\right\}$. Clearly the set $f(M)-f(K)-\bigcup_{i} q_{i}$ is a smooth 1-dimensional complex submanifold of $\mathbb{C} P^{k}$. Hence Lemma 4.2 is proved. 
Lemma 4.3. The smooth surface $f\left(V_{p}\right)$ in $\mathbb{C} P^{k}$ is a one-dimensional complex submanifold in $\mathbb{C} P^{k}$.

Proof. To prove this lemma, we only need a local argument. Let $q \in f\left(V_{p}\right)$ be an arbitrary point. Take any preimage point $p$ of $q$ in $M$. Then $d f_{p}$ is of rank two. For simplicity, we write $e_{1}$ and $e_{2}$ for $\partial_{x_{1}}$ and $\partial_{x_{2}}$ respectively. Also let $e_{3}$ be a unit vector in $\operatorname{Ker}\left(d f_{p}\right)$. The coordinate system around $p$ with respect to $\left\{e_{1}, e_{2}, e_{3}\right\}$ is denoted by $\left(x_{1}, x_{2}, x_{3}\right)$. Observe from the construction of $\exp _{p}(H)$ that we have

$$
d f_{p}=\left(\begin{array}{ccc}
f_{x_{1}}^{1} & f_{x_{2}}^{1} & f_{x_{3}}^{1} \\
\ldots & \ldots & \ldots \\
f_{x_{1}}^{k} & f_{x_{2}}^{k} & f_{x_{3}}^{k} \\
f_{x_{1}}^{1} & f_{x_{2}}^{\overline{1}} & f_{x_{3}}^{\overline{1}} \\
\ldots & \cdots & \cdots \\
f_{x_{1}}^{\tilde{k}} & f_{x_{2}}^{\tilde{k}} & f_{x_{3}}^{\tilde{k}}
\end{array}\right)_{2 k \times 3}=\left(\begin{array}{ccc}
f_{x_{1}}^{1} & f_{x_{2}}^{1} & 0 \\
\ldots & \ldots & \ldots \\
f_{x_{1}}^{k} & f_{x_{2}}^{k} & 0 \\
f_{x_{1}}^{1} & f_{x_{2}}^{\overline{1}} & 0 \\
\ldots & \cdots & \ldots \\
f_{x_{1}}^{\tilde{k}} & f_{x_{2}}^{\tilde{k}} & 0
\end{array}\right)_{2 k \times 3} .
$$

But from Corollary 2.3, for any $\alpha=1, \ldots, k$, the $\alpha$ st row and the $(\alpha+$ $k$ )st row of the last matrix in (17) are perpendicular to each other and have the same length. Hence, we can write

$$
\left(\begin{array}{c}
f_{x_{1}}^{\alpha} f_{x_{2}}^{\alpha} \\
f_{x_{1}}^{\tilde{\alpha}} f_{x_{2}}^{\tilde{\alpha}}
\end{array}\right)=\lambda_{\alpha}\left(\begin{array}{cc}
\sin \theta_{\alpha} & \cos \theta_{\alpha} \\
-\cos \theta_{\alpha} & \sin \theta_{\alpha}
\end{array}\right)
$$

or

$$
\left(\begin{array}{c}
f_{x_{1}}^{\alpha} f_{x_{2}}^{\alpha} \\
f_{x_{1}}^{\tilde{\alpha}} f_{x_{2}}^{\tilde{\alpha}}
\end{array}\right)=\lambda_{\alpha}\left(\begin{array}{c}
\sin \theta_{\alpha} \cos \theta_{\alpha} \\
\cos \theta_{\alpha}-\sin \theta_{\alpha}
\end{array}\right)
$$

for some $\lambda_{\alpha}, \theta_{\alpha} \in \mathbf{R}$. Further, it is not hard to see from Theorem 2.1 that the above two cases cannot happen simultaneously. Hence if we still denote the complex structure on $C P^{k}$ by $J$, we have for the first 
case

$$
\begin{aligned}
J \cdot d f_{p}\left(e_{1}\right) & =J\left(\sum_{\alpha=1}^{k}\left(f_{x_{1}}^{\alpha} \partial_{\alpha}+f_{x_{1}}^{\tilde{\alpha}} \partial_{\tilde{\alpha}}\right)\right) \\
& =J\left(\sum_{\alpha=1}^{k} \lambda_{\alpha}\left(\sin \theta_{\alpha} \partial_{\alpha}-\cos \theta_{\alpha} \partial_{\tilde{\alpha}}\right)\right) \\
& =\sum_{\alpha=1}^{k} \lambda_{\alpha}\left(\sin \theta_{\alpha} \partial_{\tilde{\alpha}}+\cos \theta_{\alpha} \partial_{\alpha}\right) \\
& =\sum_{\alpha=1}^{k}\left(f_{x_{2}}^{\alpha} \partial_{\alpha}+f_{x_{2}}^{\tilde{\alpha}} \partial_{\tilde{\alpha}}\right) \\
& =d f_{p}\left(e_{2}\right) .
\end{aligned}
$$

Therefore

$$
J \cdot d f_{p}\left(e_{2}\right)=J^{2} \cdot d f_{p}\left(e_{1}\right)=-d f_{p}\left(e_{1}\right) .
$$

Similarly, for the second case,

$$
J \cdot d f_{p}\left(e_{1}\right)=-d f_{p}\left(e_{2}\right), J \cdot d f_{p}\left(e_{2}\right)=d f_{p}\left(e_{1}\right) .
$$

Since $d f_{p}\left(e_{1}\right)$ and $d f_{p}\left(e_{2}\right)$ are linearly independent in $T_{f(p)} f\left(V_{p}\right)$, and $T_{f(p)} f\left(V_{p}\right)$ is two-dimensional, we obtain that $T_{f(p)} f\left(V_{p}\right)$ is $J$-invariant. Thus $f\left(V_{p}\right)$ is a one-dimensional complex submanifold in $\mathbb{C} P^{k}$ because $T_{p} f\left(V_{p}\right)$ is $J$-invariant for any point $p \in M$ of rank two. This proves Lemma 4.3.

From Lemma 4.2 and Lemma 4.3 it follows that $f(M)-f(K)-\bigcup_{i} q_{i}$ is a smooth 1-dimensional complex submanifold in $\mathbb{C} P^{k}$.

Next it is not hard to estimate the size of the image of the points of rank zero. In fact, we have

Lemma 4.4. The $\alpha$-dimensional Hausdorff measure $H^{\alpha}(f(K))$ is zero for any $\alpha>0$.

Proof. To see this, we recall a theorem of Sard ([20, Theorem 2]) which asserts that if the points of the set $A$ are all of rank 0 , $s>0$ and $f$ is of class $C^{q}, q \geq 1$, then $f(A)$ is $s / q$-null, i.e., the $s / q$-dimensional Hausdorff measure is 0 . Now our map $f$ is smooth, hence the $\alpha$-dimensional Hausdorff measure of $K$ is 0 for any $\alpha>0$. This completes the proof of Lemma 4.4.

Lemma 4.5. The image set $f(M)$ of $M$ under $f$ is an algebraic subvariety in $\mathbb{C} P^{k}$. In fact, $f(M)$ is an algebraic curve in $\mathbb{C} P^{k}$. 
Proof. By Lemma 4.2 and Lemma 4.3, $f(M)-f(K)-\bigcup_{i} q_{i}$ is a 1dimensional complex submanifold in $\mathbb{C} P^{k}$, hence $f(M)-f(K)-\bigcup_{i} q_{i}$ is open in $f(M)$. This implies that $\bigcup_{i} q_{i} \cup f(K)$ is closed in $f(M)$. Since $f$ is smooth and $M$ is compact, $f(M)$ is a compact subset in $\mathbb{C} P^{k}$ which in turn implies that $\bigcup_{i} q_{i} \cup f(K)$ is closed in $\mathbb{C} P^{k}$. By Lemma 4.4, the $\alpha$-dimensional Hausdorff measure

$$
H^{\alpha}\left(\bigcup_{i} q_{i} \cup f(K)\right) \leq H^{\alpha}\left(\bigcup_{i} q_{i}\right)+H^{\alpha}(f(K))=0
$$

for any $\alpha>0$. Now $f(M)-f(K)-\bigcup_{i} q_{i}$ is a closed 1-dimensional analytic variety of $\mathbb{C} P^{k}-f(K)-\bigcup_{i} q_{i}$. Recall an extension theorem of Shiffman [23], which is a generalization of Remmert-Stein and Bishop extension theorems, states that if $E \subset M$ is a closed set of Hausdorff $(2 t-1)$-measure zero and $U$ is a pure $t$-dimensional analytic subvariety in $M-E$ where $M$ is a complex manifold, then the topological closure $\bar{U}$ is an analytic subvariety in $M$. Now by taking $\alpha$ to be 1 and applying Shiffman's extension theorem, we conclude that the topological closure of $f(M)-f(K)-\bigcup_{i} q_{i}$ is an analytic subvariety in $\mathbb{C} P^{k}$. But $f(M)=$ $f(M)-f(K)-\cup_{i} q_{i}$. The Chow's theorem implies that $f(M)$ is an algebraic subvariety in $\mathbb{C} P^{k}$. Hence $f(M)$ is an algebraic curve since it is of one dimension. This finishes the proof of Lemma 4.5.

Remark 2. Since the singular locus $\mathcal{S}$ of an analytic subvariety of $\mathbb{C} P^{k}$ is again an analytic subvariety of $\mathbb{C} P^{k}, \mathcal{S}$ is a 0-dimensional subvariety of $\mathbb{C} P^{k}$. Clearly, any limit point of $\mathcal{S}$ belongs to $f(M)$ since $f(M)$ is closed, and hence belongs to $\mathcal{S}$ since the smooth points of the algebraic subvariety $f(M)$ form an open set in $f(M)$. So $\mathcal{S}$ is compact and therefore consists of finitely many points (see [12, p. 91]).

Remark 3. It is easy to see by the Wirtinger theorem, the area of $f(M)$ is finite (see for example [11]).

Hence Theorem 4.1 is proved.

As indicated by Theorem 4.1, we know that the image set is a smooth surface away from finitely many points, if there exist any. In fact, the stable harmonic map behaves nicely away from these singular points. We shall prove

Theorem 4.6. Let $M$ be a smooth compact manifold of three dimensions without boundary. Let $f$ be a non-constant stable harmonic map from $M$ into $\mathbb{C P}^{k}$ with the Fubini-Study metric. Then $f$ is a harmonic morphism from $M \backslash f^{-1}(\mathcal{S})$ to $f(M \backslash \mathcal{S})$, where $\mathcal{S}$ is the singular locus 
of $f(M)$.

First, we recall that a smooth map $u:(M, g) \rightarrow(N, h)$ is called horizontally weakly conformal if at each point $p \in M$

$$
g^{i j} \frac{\partial u^{\alpha}}{\partial x^{i}} \frac{\partial u^{\beta}}{\partial x^{j}}=\lambda(p) h^{\alpha \beta} .
$$

Note that $u$ is horizontally weakly conformal is equivalent to that at each point $p \in M$ either $d u_{p}=0$ or $d u_{p}$ maps the horizontal space $H_{p}=\left(\operatorname{Ker}\left(d u_{p}\right)\right)^{\perp}$ conformally onto $T_{u(p)} N$.

Lemma 4.7. $f$ is horizontally weakly conformal from $M-f^{-1}(\mathcal{S})$ to $f(M)-\mathcal{S}$.

Proof. By Lemma 4.3, $f(M)-\mathcal{S}$ is a one-dimensional submanifold in $C P^{k}$. For any point $p \in M-f^{-1}(\mathcal{S})$, we can choose a complex coordinate $z^{1}$ in a small neighborhood of $f(p)$ in $f(M)-\mathcal{S}$. Then extend $z^{1}$ to a complex coordinate in a neighborhood of $f(p)$ in $\mathbb{C P} P^{k}$, and denote it by $\left(z^{1}, \ldots, z^{k}\right)$. Notice that in this coordinate, (17) simply takes the form

$$
d f_{p}=\left(\begin{array}{ccc}
f_{x_{1}}^{1} & f_{x_{2}}^{1} & 0 \\
0 & 0 & 0 \\
\cdots & \cdots & \cdots \\
0 & 0 & 0 \\
f_{x_{1}}^{\tilde{1}} & f_{x_{2}}^{\tilde{1}} & 0 \\
0 & 0 & 0 \\
\cdots & \cdots & \cdots \\
0 & 0 & 0
\end{array}\right)_{2 k \times 3} .
$$

Furthermore, we have used the normal charts around $p$ and $f(p)$ in Theorem 2.1. Now it is immediate from (18) that $f$ is horizontally weakly conformal at $p$. Since $p$ is arbitrary in $M-f^{-1}(\mathcal{S})$, this finishes the proof of Lemma 4.7 .

Lemma 4.8. $f: M-f^{-1}(\mathcal{S})-K \rightarrow f(M)-\mathcal{S}$ is harmonic.

Proof. Consider the vertical space $V_{p}=\operatorname{Ker}\left(d f_{p}\right)$ and the horizontal space $H_{p}=\left(\operatorname{Ker} d f_{p}\right)^{\perp}$ at any rank-two point $p$ in $M$. Clearly $V_{p}$ (respectively $H_{p}$ ) is the fibre at $p$ of a vector bundle $V$ (respectively $H_{p}$ ) over $M \backslash K$. Let $\left\{e_{1}, e_{2}\right\}$ be an orthonormal frame for $H$, and $e_{3}$ be a normal frame for $V$ in a neighborhood of $p$. First, we define a complex structure by $J_{p}^{H}$ on $H_{p}$. Since $J_{p}^{H}$ and $-J_{p}^{H}$ are the only two complex structures $H_{p}$ compatible with the metric, without loss of generality, 
we assume that

$$
J_{p}^{H}\left(e_{1}\right)=e_{2}, \quad J_{p}^{H}\left(e_{2}\right)=-e_{1} .
$$

For simplicity, we use $\left(x^{1}, x^{2}, x^{3}\right)$ to denote the orthonormal coordinates in a small neighborhood of $p$. We claim that $d f_{p} \cdot J_{p}^{H}=J \cdot d f_{p}$ where $J$ is the complex structure on $\mathbb{C} P^{k}$. We observe that from (18), for the first case in (18) and (19),

$$
\begin{aligned}
& d f_{p} \cdot J_{p}^{H}\left(e_{1}\right)=d f_{p}\left(e_{2}\right)=J \cdot d f_{p}\left(e_{1}\right), \\
& d f_{p} \cdot J_{p}^{H}\left(e_{2}\right)=-d f_{p}\left(e_{1}\right)=J \cdot d f_{p}\left(e_{2}\right) .
\end{aligned}
$$

Note for the second case in (18), we have $d f_{p} \cdot J_{p}^{H}=-J \cdot d f_{p}$. Hence we replace $J_{p}^{H}$ by $-J_{p}^{H}$ in this case.

On the other hand, we observe that as a harmonic map from $M$ to $\mathbb{C} P^{k}$, the tension field $\tau(f)$ of $f$ vanishes. Therefore

$$
\begin{aligned}
0 & =\tau(f) \\
& =\operatorname{Trace} \nabla d f \\
& =\left.\operatorname{Trace} \nabla d f\right|_{H_{p} \times H_{p}}+\left.\operatorname{Trace} \nabla d f\right|_{V_{p} \times V_{p}}
\end{aligned}
$$

where $\nabla d f$ is the Hessian of $f$, and $\nabla$ is the connection induced on the bundle $T^{*}(M) \otimes f^{-1} T\left(\mathbb{C} P^{k}\right)$ over $M$ by the Levi-Civita connections on $M$ and $\mathbb{C} P^{k}$. We have shown that $f$ is horizontally holomorphic into $\mathbb{C} P^{k}$. Hence the first term of the right-hand side of (20) vanishes. Thus we have from (20) that

$$
\begin{aligned}
0 & =\left.\operatorname{Trace} \nabla d f\right|_{V_{p} \times V_{p}} \\
& =\nabla_{f_{*} e_{3}}^{\mathbb{C} P^{k}} d f\left(e_{3}\right)-d f\left(\nabla_{e_{3}}^{M} e_{3}\right) \\
& =-d f\left(\nabla_{e_{3}}^{M} e_{3}\right) .
\end{aligned}
$$

since $d f$ sends $e_{3}$ to 0 . But $\nabla_{e_{3}}^{M} e_{3}$ is the mean curvature of the fibre through $p$, and it lies in the horizontal space $H_{p}$. Since $d f$ is injective on $H_{p}$, we conclude that

$$
\nabla_{e_{3}}^{M} e_{3}=0
$$

which implies that the fibre through $p$ is minimal. 
Finally, a theroem of Baird and Eells [2] (see also [10]) asserts that for a smooth map into a surface with rank 2 somewhere if it is horizontally weakly conformal and has minimal fibres, then it is harmonic. Applying this result to our map $f$, we conclude that $f: M-K-f^{-1}(\mathcal{S}) \rightarrow$ $f(M)-\mathcal{S}$ is harmonic. Hence Lemma 4.8 is proved.

Lemma 4.9. $f$ is a harmonic morphism from $M-f^{-1}(\mathcal{S})-K$ to $f(M)-\mathcal{S}$.

Proof. From the previous two lemmas, we have seen that $f$ is horizontally weakly conformal and harmonic, as a map from $M-f^{-1}(\mathcal{S})-K$ to $f(M)-\mathcal{S}$. Further, at any rank-2 point $p \in M-f^{-1}(\mathcal{S}), d f_{p}$ : $T_{p}(M) \rightarrow T_{f(p)}(f(M)-\mathcal{S})$ is surjective. Then by the result of Ishihara [13] and Fulgede [10], we conclude that $f$ is a harmonic morphism from $M-f^{-1}(\mathcal{S})-K$ to $f(M)-\mathcal{S} \subseteq \mathbb{C} P^{k}$. This completes the proof of Lemma 4.9.

Proof of Theorem 4.6. According to Lemma 4.9, $f$ is a harmonic morphism at all rank-2 points in $M-f^{-1}(\mathcal{S})$ into $f(M)-\mathcal{S}$. Further, for any point $q$ in $K$ but not in $f^{-1}(\mathcal{S}), f(q)$ is a smooth point on the smooth surface $f(M)-\mathcal{S}$. Since $\mathcal{S}$ is closed in $f(M), f^{-1}(\mathcal{S})$ is closed in $M$. So $f^{-1}(\mathcal{S}) \cup K$ is closed in $M$, and $M-f^{-1}(\mathcal{S})-K$ is open. Next, we claim that the set $M-f^{-1}(\mathcal{S})-K$ is dense in $M$. If this is not true, then there is an connected open subset $W$ of $M$, which is contained in $f^{-1}(\mathcal{S}) \cup K$. Since $\mathcal{S}$ consists of finitely many points, we can take $W$ small enough such that $f(W)$ contains at most one point $y$ of $\mathcal{S}$. Then we consider the following two cases. Case 1: If there is a point in $W$ but not in $K$, then the rank of $f$ at this point is two. Hence there is a small open neighborhood of the point contained in $W-K$, and $f$ maps this neighborhood to the single point $y$. Case 2: Every point in $W$ is a point in $K$, then $f$ maps $W$ to a single point because the rank of $f$ is 0 at any point in $K$ and $W$ is connected. In both cases, Aronszajn's unique continuation theorem [1] implies that $f$ is a constant map. Hence $M-f^{-1}(\mathcal{S})-K$ is open and dense. Therefore we can take a sequence of points $\left\{q_{i}\right\}$ in $M-f^{-1}(\mathcal{S})-K$ such that $\lim q_{i}=q$. By Lemma 4.7 and Lemma $4.8, f$ is harmonic and weakly horizontally conformal at each $q_{i}$. Further, since $f(q)$ is a smooth point in $f(M), f$ is a smooth map from a small neighborhood of $q$ to a small neighborhood in $f(M)-\mathcal{S}$ of $f(q)$. Hence $f$ is also harmonic and weakly horizontally conformal at $q$ by approximation. Therefore, $f$ is a harmonic morphism from $M-f^{-1}(\mathcal{S})$ to $f(M)-\mathcal{S}$. This finishes the 
proof of Theroem 4.6.

\section{Obstruction on the existence of stable harmonic map from $M^{3}$ into $\mathbb{C} P^{k}$}

In this section, we will see that there exists a topological obstruction for existence of smooth stable harmonic mappings from compact 3manifold into the complex projective spaces with the standard FubiniStudy metric. Indeed, we have

Theorem 5.1. Let $f$ be a non-constant smooth stable harmonic mapping from a compact manifold of three dimensions without boundary into $\mathbb{C P}^{k}$ with the standard Fubini-Study metric. Then $M$ is a Seifert fibre space.

Proof. If $k=1$, we have shown that $M^{3}$ is a Seifer fibre space if there is a non-constant stable harmonic map into $\mathbb{C} P^{1}$. For $k \geq 2$, since $f(M)$ is an algebraic curve in $\mathbb{C} P^{k}$, we use the following standard method from algebraic geometry (see [11, p. 168]). Choose a generic $(k-2)$-plane $\mathbb{C} P^{k-2}$ in $\mathbb{C} P^{k}$ such that $\mathbb{C} P^{k-2}$ is disjoint from $f(M)$. Let $\mathbb{C} P^{1}$ be a complementary 1-plane. We choose homogeneous coordinates $X=\left[X_{0}, \ldots, X_{k}\right]$ on $\mathbb{C} P^{k}$ such that $\mathbb{C} P^{k-2}$ is given as $X_{0}=X_{1}=0$ and $\mathbb{C} P^{1}$ as $X_{2}=\ldots X_{k}=0$. Consider the projection of $\mathbb{C} P^{k}$ to $\mathbb{C} P^{1}$, projecting from $\mathbb{C} P^{k-2}$

$$
\pi\left(\left[X_{0}, \ldots, X_{k}\right]\right)=\left[X_{0}, X_{1}\right] .
$$

As shown in [11], $\pi: f(M) \rightarrow \mathbb{C P}$ is a finite sheeted branched cover of $\mathbb{C} P^{1}$, at least away from the singular locus $\mathcal{S}$. Hence $\left.\pi\right|_{f(M) \backslash \mathcal{S}}$ is holomorphic into $\mathbb{C} P^{1}$ which in turns implies that $\left.\pi\right|_{f(M) \backslash S}$ is a harmonic morphism into $\mathbb{C} P^{1}$.

Now we take the composition of $f$ with $\pi$. We see that $\pi \circ f$ is a smooth map from $M$ to $\mathbb{C} P^{1}$ for being the composition of two smooth maps. Further, $\pi \circ f$ restricts to $M \backslash f^{-1}(\mathcal{S})$ is a harmonic morphism because the composition of two harmonic morphisms is again a harmonic morphism [10]. Then it is easy to see that in fact $\pi \circ f$ is a harmonic morphism at every point on $M$ by approximation, as we have done before. Further, $\pi \circ f$ is not constant because $\left.\pi\right|_{f(M) \backslash S}$ is a finite sheeted branched cover over $\mathbb{C} P^{1}$.

Finally, we recall a result of P. Baird and J.C. Wood which states that if there exists a non-constant harmonic morphism from a compact 
3-manfold to a Riemann surface, then the compact 3-manifold is a Seifert fibre space [3]. Hence Theorem 5.1 is proved.

The following Liouville type result is just Theroem 5.1 in disguise.

Theorem 5.2. Let $M$ be a smooth compact 3-manifold which is not a Seifert fibre space. Assume that there is a smooth stable harmonic map f from $M$ into $\mathbb{C P}^{k}$ with the Fubini-Study metric. Then $f$ is constant.

Remark. The Liouville type results for smooth stable harmonic maps into the standard sphere $S^{n}$ for $n \geq 3$ were obtained by Y.L. Xin [25] for compact manifolds and by Scheon-Uhlenbeck [21] for $\mathbf{R}^{m}$.

\section{References}

[1] N. Aronszajn, A unique continuation theorem for solutions of elliptic partial differential equations of second order, J. Math. Pures Appl. 36 (1957) 235-249.

[2] P. Baird \& J. Eells, A conservation law for harmonic maps, Lecture Notes in Math., Vol. 894, Springer, Berlin, 1981, 1-25.

[3] P. Baird \& J.C. Wood, Harmonic morphisms, Seifert fibre spaces and conformal foliations, Proc. London Math. Soc. (3) 64 (1992) 170-196.

[4] E. Bishop, Conditions for the analyticity of certain sets, Michigan J. Math. 11 (1964) 289-304.

[5] D. Burns, F. Burstall, P. de Bartolomeis \& J. Rawnsley, Stability of harmonic maps of Kähler manifolds, J. Differential Geom. 30 (1989) 579-594.

[6] D. Burns \& P. de Bartolomeis, Applications harmoniques stables dans $P^{n}$, Ann. Sci. École Norm. Sup. (4) 21 (1988) 159-187.

[7] J. Carlson \& D. Toledo, Harmonic mapping of Kähler manifolds to locally symmetric spaces, Inst. Hautes Études Sci. Publ. Math., No. 69 (1989).

[8] J.Y. Chen, Stable harmonic maps into $S^{2}$, Preprint, 1993.

[9] C. Constantinescu \& A. Cornea, Compactifications of harmonic spaces, Nagoya Math. J. 25 (1965) 1-57.

[10] B. Fuglede, Harmonic morphisms between Riemannian manifolds, Ann. Inst. Fourier (Grenoble) 28-1 (1978) 107-144. 
[11] P. Griffiths \& J. Harris, Princeples of Algebraic Geometry, John Wiley, New York, 1978.

[12] R.C. Gunning, Introduction to holomorphic functions of several variables, Vol II: local theorey, Wadsworth\&Brooks/Cole, 1990.

[13] T. Ishihara, A mapping of Riemannian manifolds which preserves harmonic functions, J. Math. Kyoto Univ. 19 (1979) 169-174.

[14] C.G.J. Jacobi, Über Eine Particuläre Lösing der Partiellen Differential Gleichung $\delta^{2} v / \delta x^{2}+\delta^{2} v / \delta y^{2}+\delta^{2} v / \delta z^{2}=0$, J. Riene Angew. Math., 36 (1847) 113-134.

[15] S. Kobayashi \& Nomizu, Foundations of Differential Geometry, Vol. 2, Interscience, New York, 1969.

[16] H.B. Lawson \& J. Simons, On the stable currents and their application to global problems in real and complex geometry, Ann. of Math. (2) 98 (1973) 427-450.

[17] P.F. Leung, On stability of harmonic maps, Lecture Notes in Math., Vol. 949, Springer, Berlin, 1982, 122-129.

[18] A. Lichnerowicz, Applications harmoniques et variétés kähleriennes, Sympos. Math. 3 (1970) 341-402.

[19] Y. Ohnita \& S. Udagawa, Stable harmonic maps from Riemann surfaces to compact Hermitian symmetric spaces, Tokyo J. Math. 10 (1987) 385-390.

[20] A. Sard, Image of critical sets, Ann. of Math. 68 (1958) 247-259.

[21] R. Scheon \& K. Uhlenbeck, Regularity of minimizing harmonic maps into the sphere, Invent. Math. 78 (1984).

[22] P. Scott, The geometries of 3-manifolds, Bull. London Math. Soc. 15 (1983) 401-487.

[23] B. Shiffman, On the removal of singularities of analytic sets, Michigan J. Math. 15 (1968) 111-120.

[24] Y.T. Siu \& S.T. Yau, Compact Kähler manifolds of positive bisectional curvature, Invent. Math. 59 (1980) 189-204.

[25] Y.L. Xin, Some results on stable harmonic maps, Duke Math. J. 47 (1980) 609-613.

[26] J.C. Wood, Harmonic morphism, foliations and Gauss maps, Contemp. Math. 49 (1986) 145-184. 
STABLE HARMONIC MAPS

UNIVERSity of CALIForNia, IRVINE 